Research Paper

\title{
Response Rates as Predictors of Overall Survival: A Meta-Analysis of Acute Myeloid Leukemia Trials
}

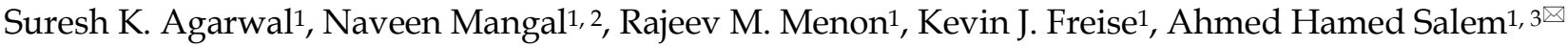 \\ 1. Abbvie, Inc., North Chicago, IL, USA; \\ 2. Center for Pharmacometrics and Systems Pharmacology, University of Florida, Orlando, FL, USA; \\ 3. Department of Clinical Pharmacy, Faculty of Pharmacy, Ain Shams University, Cairo, Egypt. \\ $\triangle$ Corresponding author: Ahmed Hamed Salem, PhD, AbbVie Inc., Department R4PK, Building AP31-3, 1 North Waukegan Road, North Chicago, IL 60064 \\ Telephone: +1 847- 938-0776 Facsimile: +1 847-938-5193 Email: ahmed.salem@abbvie.com \\ (c) Ivyspring International Publisher. This is an open access article distributed under the terms of the Creative Commons Attribution (CC BY-NC) license \\ (https://creativecommons.org/licenses/by-nc/4.0/). See http://ivyspring.com/terms for full terms and conditions.
}

Received: 2016.12.09; Accepted: 2017.03.31; Published: 2017.06.03

\begin{abstract}
Background: Response rates such as overall response rate (ORR), complete response (CR) and complete response with incomplete blood recovery (CRi) can be evaluated in a much shorter period of time than overall survival (OS), potentially accelerating decision making during drug development. The objective of this work was to evaluate the relationship between ORR, CR, CRi or better $(C R i+C R)$ rates and median OS to determine whether response rates could be used as predictors of median OS in acute myeloid leukemia (AML).

Methods: A review of published literature was conducted to identify relevant AML clinical trials. Weighted linear regression was performed with various linearizing transformations of response rates and median OS. Covariates of interest were evaluated using a forward inclusion, backward elimination covariate model building procedure at $\alpha=0.01$ and $\alpha=0.005$, respectively.

Results: Twenty trials involving 26 cohorts were included in the meta-analysis. Azactidine treatment was a significant predictor with longer OS compared to decitabine or low dose cytarabine for a given response rate $(P<0.005)$. Linear regression analysis indicated that the correlation of both CRi or better rates and CR rates with median OS was higher than that of ORR with median OS. The final model showed a strong correlation between CRi or better rates and median OS $\left(R^{2}=0.66\right)$.

Conclusion: Significant correlation between CRi or better rates and median OS in AML highlights the potential for CRi or better rate, in addition to CR rate, to serve as surrogate markers for median OS.
\end{abstract}

Key words: Acute myeloid leukemia, surrogate endpoints, overall survival, response rates, azacitidine, meta-analysis.

\section{Introduction}

Overall survival (OS) is widely used as a primary endpoint for the approval of oncology drugs [1]. Measurement of OS is relatively easy, precise and devoid of subjective assessment as there can be only 2 mutually exclusive outcomes (surviving or non-surviving). However, its evaluation requires long patient follow-up time in a clinical trial, increasing the time and resources required for developing novel therapies. This can also hamper the ability to design future trials, including selection of optimal starting doses. In some malignancies, response rate-based outcomes such as complete response (CR), partial response (PR) or overall response rate (ORR) can be used as surrogate endpoints, which can be measured in a much shorter period of time compared to OS, potentially making a case for accelerated drug approval by regulatory agencies. From 2005 to 2012, the United States Food and Drug Administration (US FDA) approved 41 indications for oncology drugs, $84 \%$ of which were based on surrogate endpoints such 
as progression free survival (PFS) and CR [2]. Continuing the trend, 16 out of 17 indications for oncology drugs approved in 2014 to mid-2015 were based on surrogate endpoints, with ORR being the most common surrogate endpoint [3].

Currently there are no drugs approved in the United States for elderly patients who are newly diagnosed with AML and considered ineligible for standard induction therapy. However, low-intensity treatment options such as azacitidine, decitabine and low dose cytarabine (LoDAC) are currently recommended by the National Comprehensive Cancer Network (NCCN) guidelines for patients aged $\geq 60$ years. The relationship between response rates and OS has not been assessed across AML clinical trials, although trends have been observed in individual studies. In a recently reported Phase 2 trial investigating pracinostat and azacitidine in elderly patients with newly diagnosed AML deemed unsuitable for intensive therapy, OS was highly correlated with CR. Two $(10 \%)$ of the 21 patients with CR died, compared with $20(69 \%)$ of the 29 patients without CR; median OS was not reached in those with $\mathrm{CR}$, whereas a median OS of 7.6 months was observed in those without $C R(P<0.0001)$ [4]. In another Phase 2 trial of treatment-naïve elderly AML patients, Kantarjian et al. reported a median OS of 19.1 and 15.8 months for patients with CR and CRi + CRp (complete remission with incomplete blood count recovery + complete remission with incomplete platelet recovery), respectively. On the other hand, patients who did not achieve a CR/CRi/CRp had a median OS of 3.1 months; significantly shorter than the median in patients who did achieve CR/CRi/CRp $(\mathrm{P}<0.0001)$ [5].

The primary objective of this work was to determine the relationship between response rate-based outcomes (ORR, CRi or better, and CR) and median OS in elderly AML patients to assess the potential of response rates to serve as predictors for median OS.

\section{Methods}

\section{Trial Selection}

The primary source of information for this database was PubMed articles published in English between the years 2004 and 2016. During the PubMed search, the patient subfilters used were "acute myeloid leukemia," "newly diagnosed," and "'azacitidine, decitabine or cytarabine," and the study design and publication type subfilters were "clinical trial," "monotherapy," and "primary publication." Azacitidine, decitabine and cytarabine were chosen, as these drugs are currently recommended by NCCN guidelines for use in elderly patients who are unfit for standard induction therapy. A trial was included in the database if at least one cohort of the study evaluated the use of azacitidine, decitabine or LoDAC and had at least 1 primary or secondary outcome reported as ORR or OS. Other information that was collected includes trial design, sample size, treatment, AML type, percentage of males, age, baseline bone marrow blast percent, baseline absolute neutrophil counts, and Eastern Cooperative Oncology Group (ECOG) scores, if reported. A trial was excluded from the database if the study type was not relevant, including retrospective studies, reviews, meta-analyses, case-reports or cost-analyses.

\section{Meta-analysis Methodology}

Descriptive statistics were performed to summarize the characteristics of the cohorts selected for the analysis. To improve the linearity of the relationship, various transformations of response rates (e.g. logit, arcsine) and median OS data (e.g. logarithm, reciprocal, square root, cube root) were tested. Linear regression weighted by sample size was then performed to determine the correlation between response rates $(\mathrm{CR}, \mathrm{CRi}$ or better, ORR) and median OS on a transformed scale using $\mathrm{R}$ v.3.2.1 (http://www.r-project.org/). Effects of covariates such as median age, percentage of males enrolled, treatment, percentage of patients with ECOG scores of $\leq 1$, and median bone marrow blast percentage were evaluated using a stepwise forward inclusion, backward elimination model building procedure at Type 1 error rates of $\alpha=0.01$ and $\alpha=0.005$, respectively. Adjusted coefficient of determination $\left(R^{2}\right)$ and model diagnostic plots were used to assess the performance of the models. The model results were back-transformed to the original scale for plotting the relationship between response rates and median OS.

\section{Results}

Overall, 33 trials were included in the database following the outlined inclusion and exclusion criteria. Using the established database, a trial was included in the meta-analysis if it had both response rates and median OS reported in at least one cohort of the study (Figure 1). Twenty trials (6 of which were randomized) involving 26 cohorts were included in the meta-analysis. A detailed description of characteristics of cohorts included in the analysis is presented in Table 1. In brief, the median age of patients ranged from 66 to 78 years and the median and range of outcomes were as follows: ORR $28 \%$ (6-68\%); CRi 3\% (0-17\%); CR 18\% (5-57\%); and OS 7.7 months (3.0-18.9 months). Half of the cohorts (50\%) included azacitidine alone or in combination, with 
decitabine being the second most used treatment option $(26.9 \%)$. ECOG scores and median bone marrow blast percentage were reported in approximately two-thirds of the cohorts. The percentage of patients with ECOG score $\leq 1$ ranged from 52.2 to 82.2 , with a median value of 75.5 , which was imputed for cohorts in trials not reporting ECOG scores. Similarly, the median bone marrow blast percentage ranged from $25 \%$ to $72 \%$ with a median value of $46 \%$ imputed for cohorts in trials in which it was not reported.

\section{Meta-analysis}

Logit of response rates and logarithm of median OS were the best transformations of the data. The linear regression coefficient was used to assess the strength of the relationship between response rates (ORR, CR, and CRi or better) and median OS. The correlation between $\mathrm{CRi}$ or better or $\mathrm{CR}$ rates and median OS was higher $\left(\mathrm{R}^{2}=0.49\right.$ and 0.48, respectively) than that of ORR and median OS $\left(R^{2}=0.45\right)$. Azacitidine treatment was found to be a significant covariate in the model $(\mathrm{P}<0.005)$, with higher median OS in cohorts receiving treatment with azacitidine at a given CRi or better rate compared with those receiving decitabine or LoDAC. For example, at a CRi or better rate of $30 \%$, the estimated median OS was approximately 3 months (95\% CI: 2.2 3.8 months) longer for the azacitidine cohort compared to the non-azacitidine cohorts. The final model showed significant correlation between CRi or better rates and median OS $\left(\mathrm{R}^{2}=0.66\right.$, Figure 2$)$. Other covariates, such as median age, percentage of males, ECOG scores, and bone marrow blast percentage were not found to be statistically significant.

Table 1. Summary of the characteristics of the cohorts included in the meta-analysis

\begin{tabular}{ll}
\hline Characteristics & Number of cohorts (\%) \\
\hline Total number of cohorts & $26(100 \%)$ \\
Publication year & \\
$\quad(2006-2012)$ & $10(38.5)$ \\
$\quad(2013-2016)$ & $16(61.5)$ \\
Median age & \\
$\quad<75$ years & $16(61.5)$ \\
$\quad \geq 75$ years & $10(38.5)$ \\
Percentage of males & \\
$\quad<50$ & $4(15.4)$ \\
$\geq 50$ & $21(74)$. \\
$\quad$ Not reported & $1(3.8)$ \\
Percentage of patients with ECOG scores of $\leq 1$ & \\
$\quad<75$ & $7(26.9)$ \\
$\quad \geq 75$ & $10(38.5)$ \\
$\quad$ Not reported & $9(34.6)$ \\
Median bone marrow blast percentage & \\
$\quad<50$ & $9(34.6)$ \\
$\quad \geq 50$ & $7(26.9)$ \\
$\quad$ Not reported & $10(38.5)$ \\
Treatment cohorts & \\
Azacitidine & $13(50)$ \\
Decitabine & $7(26.9)$ \\
Cytarabine & $3(11.5)$ \\
Others (Intensive chemotherapy, Supportive Care) & $3(11.5)$ \\
\hline
\end{tabular}

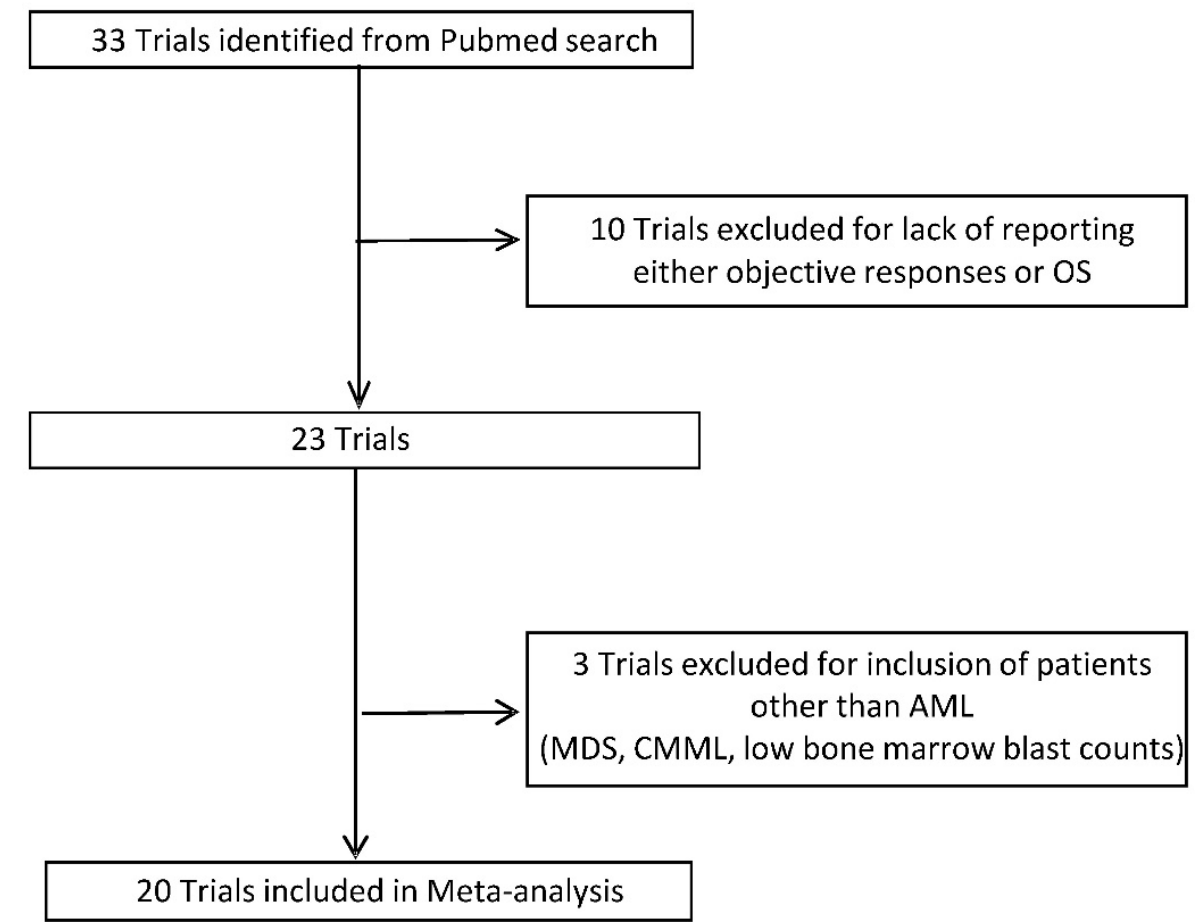

Figure 1. Selection of Trials for Analysis. OS: Overall survival; AML: Acute myeloid leukemia; MDS: Myelodysplastic syndromes; CMML: Chronic myelomonocytic. 

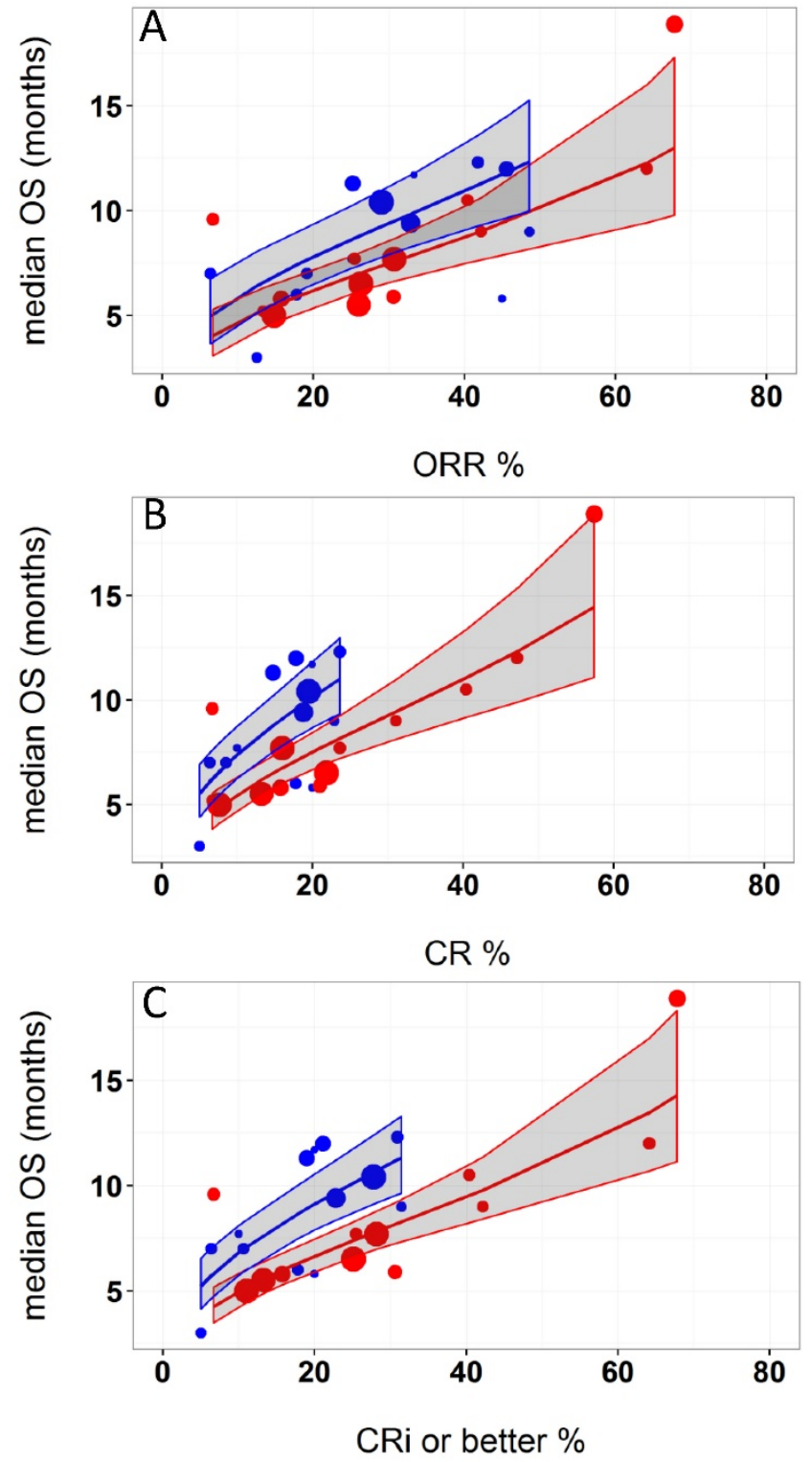

Figure 2. Relationship between $A$ ) overall response rate (ORR), B) complete response $(C R)$, and $C$ ) complete response with incomplete blood recovery (CRi) or better and median overall survival (OS) in acute myeloid leukemia. Each filled circle corresponds to a treatment cohort, with the area of the circle being proportional to its sample size. Blue lines indicate the fitted values for azacitidine cohorts and red lines indicate fitted values for cohorts other than azacitidine. Shaded regions indicate the $95 \%$ confidence intervals.

\section{Discussion}

There is an unmet medical need for better therapies for elderly AML patients; however, the use of OS as an endpoint in elderly AML patients may require 2-3 years to demonstrate survival benefit. On the other hand, response rates usually require only 6 months follow up in AML trials. Hence, the use of response rates as surrogate measures for establishing efficacy in AML trials has the potential to accelerate drug approval by approximately two years [6]. In a review of FDA experience with 47 new indications for 35 oncology products which were granted accelerated approval between 1992 and 2010, the median time between accelerated approval and regular approval of oncology products was 3.9 years allowing substantially earlier access of cancer patients to these drugs that demonstrate significant clinical benefit [7]. This potentially reduces health care costs and minimizes hospital visits.

Examples of accelerated approvals converting to regular approvals include bortezomib and carfilzomib indicated for multiple myeloma. Both drugs gained accelerated approval based on response rates (May 2003 and July 2012, respectively) and were subsequently converted to regular approvals (March 2005 and January 2016, respectively) based on survival benefit. On the other hand, gemtuzumab gained accelerated approval in May 2000 as a second-line therapy for AML in patients older than 60 years but was voluntarily withdrawn from market ten years later after it failed to demonstrate improved efficacy and acceptable toxicity in a randomized study [8]. However, additional data from 4 randomized studies has shown the efficacy of this agent in newly diagnosed AML with acceptable toxicity and its application was recently resubmitted almost seven years after withdrawal [8]. It must be noted that an accelerated approval pathway provides early access to a potential therapy for patients but does not necessarily reduce drug development costs for drug companies because companies are required to demonstrate the direct clinical/survival benefit subsequent to accelerated approval to gain regular approval.

The current work represents the first evaluation of the use of response rates as predictors of median OS, potentially accelerating the development of novel AML therapies. The analysis included clinical trials that evaluated the three commonly used drugs (azacitidine, decitabine and LoDAC) that are currently recommended by NCCN guidelines for use in elderly patients with AML who are unfit for standard induction therapy. In general, studies of single agent therapy with these drugs have shown response rates in the range of $25-30 \%$ [9-11]. It must be noted that a number of novel drugs that are being investigated in combination with these commonly used drugs were successful in increasing the response rates, especially CRi [4, 12-18]. Therefore, trials investigating these three drugs were considered to be most relevant for inclusion in our analysis to predict the potential OS of the new combination therapies.

US FDA regulations allow new drug approval to proceed via two pathways: regular and accelerated. Direct clinical benefit or an effect on an endpoint established as a surrogate must be demonstrated for regular approval. For accelerated approval, which is 
designed to provide rapid access to therapies for life-threatening diseases, an effect must be demonstrated on a surrogate measure that is "reasonably likely" to predict clinical benefit, allowing use of a surrogate before being established. Generally in leukemia, durable $\mathrm{CR}$ has been considered an established endpoint of clinical benefit if associated with less infection, bleeding or blood product support and patients with durable CR have been shown to have longer OS compared to those without CR $[1,19$, 20]. However, contradictory evidence from recent studies investigating new AML therapies show higher $\mathrm{CR}$ rates compared to control, with no improvement in survival [21, 22], suggesting remission does not always result in survival benefit. Additionally, data from less intensive treatment options, such as azacitidine in elderly patients, suggested no survival difference between subjects achieving CR and subjects achieving less stringent responses including $\mathrm{CRi}$ and PR $[23,24]$. Therefore, there is considerable interest in understanding the relationship between less stringent responses and OS and factors affecting this relationship in elderly patients. To that end, our analysis demonstrated that OS was strongly correlated with $\mathrm{CR}$ and CRi or better rates in elderly AML patients treated with low-intensity treatments. There was no difference in OS predictability between $\mathrm{CR}$ and $\mathrm{CRi}$ or better rates.

Another consideration for predicting survival was the impact of drug treatment on median OS. Notably, among the three drugs that were evaluated, azacitidine appears to achieve a longer OS compared to decitabine or LoDAC for a given response rate. One possible mechanism for longer OS with azacitidine could be the ability to administer it for a longer period of time compared to decitabine and LoDAC (median treatment cycles: azactidine - 6 to 8 cycles; decitabine/LoDAC - 2 to 5 cycles) [9-11]. Our analysis suggests that at the population level, OS predictions made using CRi or better rates are similar to those made using $\mathrm{CR}$ rates alone. However, this cannot be taken to demonstrate that the survival outcome at individual level for a patient with CRi is identical to that for a patient with CR.

In summary, the relationship between response rate outcomes and median OS was determined in elderly AML patients. The analysis demonstrated that $\mathrm{CRi}+\mathrm{CR}$ rate was a better predictor of median OS than OR rate and that the median OS was longer with azacitidine treatment compared to treatment with decitabine or LoDAC for a given response rate. These estimated relationships in AML highlights the potential for CRi or better rates, in addition to CR rate, to serve as surrogate markers for median OS and may be used to guide decisions on long-term survival using only short-term response rates in the development of new therapies for AML.

\section{Abbreviations}

ORR: overall response rate; CR: complete response; CRi: complete response with incomplete blood recovery; OS: overall survival; AML: acute myeloid leukemia; PR: partial response; US FDA: United States Food and Drug Administration; PFS: progression free survival; LoDAC: low-intensity treatment options such as azacitidine, decitabine and low dose cytarabine; NCCN: National Comprehensive Cancer Network; CRp: complete remission with incomplete platelet recovery; ECOG: Eastern Cooperative Oncology Group.

\section{Acknowledgements}

The authors would like to acknowledge AbbVie employees Francis Zaw-Myo-Htet Myasein for assembling the database and Allison Kitten for medical writing support.

\section{Competing Interests}

AbbVie participated in database preparation, data analysis, writing, reviewing and approval of the manuscript. SKA, RM, KJF, and AHS are AbbVie employees and may hold AbbVie stock and/or stock options. NM is a former intern of AbbVie.

\section{References}

1. US Food and Drug Administration Guidance for Industry. Clinical Trial Endpoints for the Approval of Cancer Drugs and Biologics. US Department of Health and Human Services, 2007. http://www.fda.gov/downloads/ Drugs/.../Guidances/ucm071590.pdf.

2. Downing NS, Aminawung JA, Shah ND, Krumholz HM, Ross JS. Clinical trial evidence supporting FDA approval of novel therapeutic agents, 2005-2012. Jama. 2014; 311: 368-77

3. Editorial. Oncology Endpoints in a Chaging Landscape. Managed Care. 2016; 1 (Suppl): 1-12. Available from: http://editiondigital.net/publication/?i= 290237\#\{"issue_id":,"page":52\}.

4. Garcia-Manero G, Atallah E, Medeiros B, Arellano M, Khaled SK, Patnaik M, et al. Final results from a phase 2 study of pracinostat in combination with azacitidine in elderly patietns with acute myeloid leukemia (AML). Blood. 2015; $126: 453$

5. Kantarjian HM, Roboz GJ, Kropf PL, Yee KWL, O'Connell CL, Tibes R, et al. Comparison of efficacy and safety results in 103 treatment-naive acute myeloid leukemia (TN-AML) patients not candidates for intensive chemotherapy using 5-day and 10-day regimens of guadecitabine (SGI-110), a novel hypomethylating agent (HMA). 57th ASH Annual Meeting and Exposition 2015 Available at: http://wwwastxcom/content/resources/ 2015_SGI-110_Oral_ASH_abst-458_Kantarjianpdf.

6. Estey E, Othus M, Lee S, Appelbaum F, Gale R. New drug approvals in acute myeloid leukemia: what's the best end point? Nature Publishing Group; 2015.

7. Johnson JR, Ning Y-M, Farrell A, Justice R, Keegan P, Pazdur R. Accelerated approval of oncology products: the food and drug administration experience. Journal of the National Cancer Institute. 2011.

8. Rowe JM, Löwenberg B. Gemtuzumab ozogamicin in acute myeloid leukemia: a remarkable saga about an active drug. Blood. 2013; 121: 4838-41.

9. Dombret H, Seymour JF, Butrym A, Wierzbowska A, Selleslag D, Jang JH, et al. International phase 3 study of azacitidine vs conventional care regimens in older patients with newly diagnosed AML with $>30 \%$ blasts. Blood. 2015; 126 : 291-9.

10. Fenaux P, Mufti GJ, Hellstrom-Lindberg E, Santini V, Gattermann N, Germing $\mathrm{U}$, et al. Azacitidine prolongs overall survival compared with conventional care regimens in elderly patients with low bone marrow blast count acute myeloid leukemia. J Clin Oncol. 2010; 28: 562-9.

11. Kantarjian HM, Thomas XG, Dmoszynska A, Wierzbowska A, Mazur G, Mayer J, et al. Multicenter, randomized, open-label, phase III trial of decitabine 
versus patient choice, with physician advice, of either supportive care or low-dose cytarabine for the treatment of older patients with newly diagnosed acute myeloid leukemia. J Clin Oncol. 2012; 30: 2670-7.

12. Fathi AT, Erba HP, Lancet JE, Stein EM, Walter RB, DeAngelo DJ, et al SGN-CD33A Plus Hypomethylating Agents: A Novel, Well-Tolerated Regimen with High Remission Rate in Frontline Unfit AML. Blood. 2015; 126: 454.

13. Visani G, Loscocco F, Fuligni F, Zuffa E, Sensi A, Zaccaria A, et al. Tosedostat Plus Low Dose Cytarabine Induces a High Rate of Responses That Can be Predicted By Genetic Profiling in Elderly AML. Blood. 2015; 126: 329.

14. Dohner H, Lubbert M, Fiedler W, Fouillard L, Haaland A, Brandwein JM, et al. Randomized, phase 2 trial of low-dose cytarabine with or without volasertib in AML patients not suitable for induction therapy. Blood. 2014; 124: 1426-33.

15. DiNardo C, Pollyea D, Pratz K, Thirman MJ, Letai A, Frattini M, et al. A phase 1b study of venetoclax (ABT-199/GDC-0199) in combination with decitabine or azacitidine in treatment-naive patients with acute myelogenous leukemia who are $\geq$ to 65 years and not eligible for standard induction therapy. Blood. 2015; $126: 327$

16. Lin TL, Strickland SA, Fiedler W, Walter RB, Hou J-Z, Roboz GJ, et al. Phase $\mathrm{Ib} / 2$ study of venetoclax with low-dose cytarabine in treatment-naive patients age $\geq 65$ with acute myelogenous leukemia. J Clin Oncol. 2016; 34: (suppl): abstr7007.

17. Agarwal SK, DiNardo CD, Potluri J, Dunbar M, Kantarjian HM, Humerickhouse RA, et al. Management of Venetoclax-Posaconazole Interaction in Acute Myeloid Leukemia Patients: Evaluation of Dose Adjustments. Clinical Therapeutics. 2017; 39: 359-67.

18. Wei A, Strickland SA, Roboz GJ, Hou J-Z, Fiedler W, Lin TL, et al. Safety and Efficacy of Venetoclax Plus Low-Dose Cytarabine in Treatment-Naive Patients Aged $\geq 65$ Years with Acute Myeloid Leukemia. Am Soc Hematology; 2016.

19. Appelbaum FR, Rosenblum D, Arceci RJ, Carroll WL, Breitfeld PP, Forman SJ, et al. End points to establish the efficacy of new agents in the treatment of acute leukemia. Blood. 2007; 109: 1810-6.

20. Freireich EJ, Gehan EA, Sulman D, Boggs DR, Frei E, 3rd. The effect of chemotherapy on acute leukemia in the human. Journal of chronic diseases. 1961; 14: 593-608.

21. Burnett AK, Russell NH, Hunter AE, Milligan D, Knapper S, Wheatley K, et al. Clofarabine doubles the response rate in older patients with acute myeloid leukemia but does not improve survival. Blood. 2013; 122: 1384-94.

22. Burnett AK, Hills RK, Hunter AE, Milligan D, Kell WJ, Wheatley K, et al. The addition of gemtuzumab ozogamicin to low-dose Ara-C improves remission rate but does not significantly prolong survival in older patients with acute myeloid leukaemia: results from the LRF AML14 and NCRI AML16 pick-a-winner comparison. Leukemia. 2013; 27: 75-81.

23. Thepot S, Itzykson R, Seegers V, Recher C, Raffoux E, Quesnel B, et al. Azacitidine in untreated acute myeloid leukemia: a report on 149 patients. Am J Hematol. 2014; 89: 410-6.

24. Pleyer L, Burgstaller S, Girschikofsky M, Linkesch W, Stauder R, Pfeilstocker $\mathrm{M}$, et al Azacitidine in 302 patients with WHO-defined acute myeloid leukemia: results from the Austrian Azacitidine Registry of the AGMT-Study Group. Annals of hematology. 2014; 93: 1825-38. 